\title{
Dynamical behaviour of multi-particle large-scale systems
}

\author{
António M. Lopes • J.A. Tenreiro Machado
}

\begin{abstract}
Collective behaviours can be observed in both natural and man-made systems composed of a large number of elemental subsystems. Typically, each elemental subsystem has its own dynamics but, whenever interaction between individuals occurs, the individual behaviours tend to be relaxed, and collective behaviours emerge. In this paper, the collective behaviour of a large-scale system composed of several coupled elemental particles is analysed. The dynamics of the particles are governed by the same type of equations but having different parameter values and initial conditions. Coupling between particles is based on statistical feedback, which means that each particle is affected by the average behaviour of its neighbours. It is shown that the global system may unveil several types of collective behaviours, corresponding to partial synchronisation, characterised by the existence of several clusters of synchronised subsystems, and global synchronisation between particles, where all the elemental particles synchronise completely.
\end{abstract}

\author{
Keywords \\ Large-scale system · Collective behaviour . \\ Dynamical interaction $\cdot$ Synchronisation
}

\section{Introduction}

A large-scale dynamical system is composed of a large number of identical individual subsystems that may interact with each other. Each elemental system has its own dynamics but, when interaction exists, the individual behaviour tends to be relaxed, and a collective behaviour emerges. Such kind of phenomenon is common in nature as well as in man-made systems [5, $6,9,19,32]$. For example, it is well known that collective behaviours emerge in colonies of birds, fish, and bees, where each individual adapts its own motion to a common pattern. In man-made transportation systems, as vehicles travelling on a highway, drivers often use social information, such as the driving behaviour of others, and adjust their own behaviours. Almost the same is observed in pedestrians to judge a safe gap in traffic. Pedestrians seem to use social information, such as the crossing behaviour of others, and follow others across the road [10]. Collective behaviours can also be observed in physical systems at thermodynamic equilibrium. For example, it is well known that above the Curie temperature, neighbouring magnetic spins in a paramagnetic material display a completely disordered state. At the Curie temperature, they suddenly align to each other, pointing toward the 
same direction, and the material becomes ferromagnetic [9].

Synchronisation is a particular collective behaviour that may arise in large-scale dynamical systems and has received considerable attention recently $[3,4,7$, $12,22,26,28,38,39]$. Synchronisation may be interpreted as a process by which individual systems adjust a given property of their motion to a common behaviour [3]. Study of synchronisation started in the 17th century with Huygens observations. He found that two weakly coupled pendulum clocks, hanging at the same beam, become phase synchronised. Synchronisation phenomena occur widely in nature and in biology. For example, populations of fireflies synchronise their flashing [29], and several intercellular coupling mechanisms lead to synchronised oscillators that govern fundamental physiological processes, such as cardiac function, respiration and circadian rhythms $[7,8,15]$. In engineering and technology, synchronisation plays an important role in secure communication systems [14, 18, 21], electric power systems [33] and robotics [16, 23], just to mention a few.

Synchronisation has been widely studied in the context of chaotic dynamical systems $[1,2,4,11-$ $13,17,20,24-28,35-39]$, mainly involving pairs of identical chaotic oscillators. In this context, synchronisation control, stability analysis, and synchronisation characterisation have been addressed by several authors $[4,12,28]$. It has been observed that different synchronisation levels may be achieved between interacting dynamical systems [3]. In complete (or identical) synchronisation, trajectories of equivalent state variables are coincident $[17,36]$. This kind of synchronisation was firstly shown for two identical chaotic systems unidirectionally coupled [26]. Generalised synchronisation refers to synchronisation between non-identical interacting systems [20,35]. Usually, in this case, complete synchronisation cannot be entirely achieved and, therefore, generalised synchronisation usually denotes a weaker synchronisation regime. In phase synchronisation, phase lock is achieved, whereas the amplitudes of equivalent trajectories remain uncorrelated [1, 25]. In lag synchronisation, both the phases and the amplitudes of equivalent trajectories are locked, but a time lag exists. This means that lag synchronisation is some state between complete and phase synchronisation [27]. Intermittent lag synchronisation is observed when the synchronising systems are generally lag synchronised, however, during some time periods, intermittent nonsynchronous behaviour may occur [2, 27]. Imperfect phase synchronisation can be characterised by phase slips during phase synchronisation regimes [37]. Finally, in almost synchronisation, the errors between trajectories of a subset of equivalent state variables are asymptotically bounded [11].

Two main coupling configurations have been considered in the literature, namely, unidirectional (or master-slave) and bidirectional [3]. In the former, considering a two dynamical systems case, the master influences (drives) the slave, but its motion is not affected by it. In the latter case, both systems influence each other and adjust their motions accordingly.

With respect to large-scale systems composed of identical subsystems the two main types of synchronised behaviours are global and cluster synchronisation [34]. Global synchronisation can be considered as a generalisation of complete synchronisation. Systems composed of non-identical subsystems might show all types of generalised synchronisation observed in pairs of coupled oscillators.

In this paper a large-scale system constituted by several coupled subsystems, or particles, is considered. The particles are located randomly in space. Moreover, they are identical to each other, that is, they are described by the same differential equations, but present, possibly, different dynamic parameters and initial conditions $[30,31]$. Coupling between a large-number of particles based on statistical feedback, which means that each particle is affected by the average behaviour of its neighbours, and several measures for analysing global and partial synchronisation are proposed. It is shown that the global system may unveil several collective behaviours, corresponding to partial synchronisation, characterised by the existence of several clusters of synchronised oscillators, and global synchronisation between particles, where all the elemental particles synchronise completely.

Bearing these ideas in mind, this paper is organised as follows. Section 2 formulates the system dynamics. Section 3 presents simulation results and analyses the system behaviour. Conclusions are drawn in Section 4.

\section{System dynamics}

A system consisting of $n$ identical components, or elemental particles, located on a two-dimensional space 
is considered. The location of each elemental particle $i, i=1, \ldots, n$, is given by the vector $\mathbf{x}_{p i}=\left[x_{p i} y_{p i}\right]$ and the corresponding dynamics is represented by

$$
\begin{aligned}
& \dot{x}_{1 i}=x_{2 i} \\
& \dot{x}_{2 i}=-\frac{K_{i}}{M_{i}} x_{1 i}
\end{aligned}
$$

where $x_{1 i}$ and $x_{2 i}$ represent the particle's state variables and $M_{i}, K_{i}$ are particles' parameters, respectively.

The overall system dynamics may the described by $2 n$ state variables, corresponding to the state variables of all elemental particles. Nevertheless, as we are mainly interested in the collective behaviour of the elemental components, two new variables are defined, namely $x_{1 \text { sist }}$ and $x_{2 \text { sist }}$, according to (2) and (3), representing the average values of the particles' first and second state variables, respectively.

$$
\begin{gathered}
1 \\
x_{\text {Isist }}=\frac{1}{n} \sum_{i=1}^{n} x_{1 i} \\
x_{2 \text { sist }}=\frac{1}{n} \sum_{i=1}^{n} x_{2 i}
\end{gathered}
$$

Each particle, $i$, interacts with neighbour particles, $j$, that are closer to $i$ than a limit threshold, $r$. For distance between particles $i$ and $j, d_{i j}$, we adopted the Euclidean measure. Interaction is given by expression (4),

$$
\begin{aligned}
& \dot{x}_{1 i}=x_{2 i}+G \cdot\left(a v_{1}-x_{1 i}\right) \\
& \dot{x}_{2 i}=-\frac{K_{i}}{M_{i}} x_{1 i}+G \cdot\left(a v_{2}-x_{2 i}\right)
\end{aligned}
$$

where $a v_{1}$ and $a v_{2}$ represent the average values of the first and second state variables of particles $j$ (the neighbours of $i$ ), respectively, given by

$$
\begin{aligned}
& a v_{1}=\frac{1}{|S|} \sum_{j \in S} x_{1 j} \\
& a v_{2}=\frac{1}{|S|} \sum_{j \in S} x_{2 j}
\end{aligned}
$$

and $|S|$ represents the cardinal number of set $S$, given by (7). The constant $G$ represents the interaction strength or gain.

$$
\begin{aligned}
& S=\left\{j: d_{i j} \leq r, j \neq i\right\} \\
& d_{i j}=\sqrt{\left(x_{p i}-x_{p j}\right)^{2}+\left(y_{p i}-y_{p j}\right)^{2}}
\end{aligned}
$$

\section{System simulation and behaviour analysis}

For the system simulation, it is considered that the overall system consists of $n=100$ elemental particles. They are randomly located on the $\mathbf{x y}$ space, according to a uniform probability distribution (9), where $0 \leq \mathrm{rnd} \leq 1$ is a random number generator.

$$
\begin{aligned}
& x_{p i}=100 \cdot \text { rnd } \\
& y_{p i}=100 \cdot \text { rnd }
\end{aligned}
$$

The particles are characterised by different dynamical parameters and initialised with distinct conditions. The particles have the same parameter, $M_{i}=1$, but $K_{i}$ is random, according to a uniform probability distribution, in the interval $1 \times 10^{6} \leq K_{i} \leq 4 \times 10^{6}$. This means that the elemental particles can $/$ oscillate at an angular frequency $1 \times 10^{3} \leq \omega_{i}=K_{i} / M_{i} \leq$ $2 \times 10^{3} \mathrm{rad} / \mathrm{s}$, in the absence of interaction. The particles' initial state, $x_{1 i}(0)$ and $x_{2 i}(0)$, is also generated randomly, following a uniform distribution in the intervals, $-2 \leq x_{1 i}(0) \leq 2$ and $-5 \leq x_{2 i}(0) \leq 5$, respectively. Coupling gain is established as $G=10^{4}$.

The motion of the elemental particles is calculated by means of a Runge-Kutta Fourth order numerical integration algorithm, with fixed step size, $d t=10^{-4} \mathrm{~s}$, for a simulation time window, $T_{w}=2 \mathrm{~s}$.

For a given distribution of particles, in the $\mathbf{x y}$ plane, and a given distance threshold, $r$, there is a certain number of particles' interaction clusters. Each interaction cluster consists of particles that interact between themselves. Particles belonging to different interaction clusters do not have any type of interaction. The clusters are computed using agglomerative hierarchical clustering analysis. Firstly, the Euclidean distance between each pair of particles is computed. In a second step, based on that metric, the single linkage criterion is used to create the hierarchical cluster tree and, for a given threshold value measured by the Euclidean distance, the cluster tree is pruned and the clusters created.

Figure 1 represents a particular random distribution of $n=100$ particles on the plane, according to (9). 


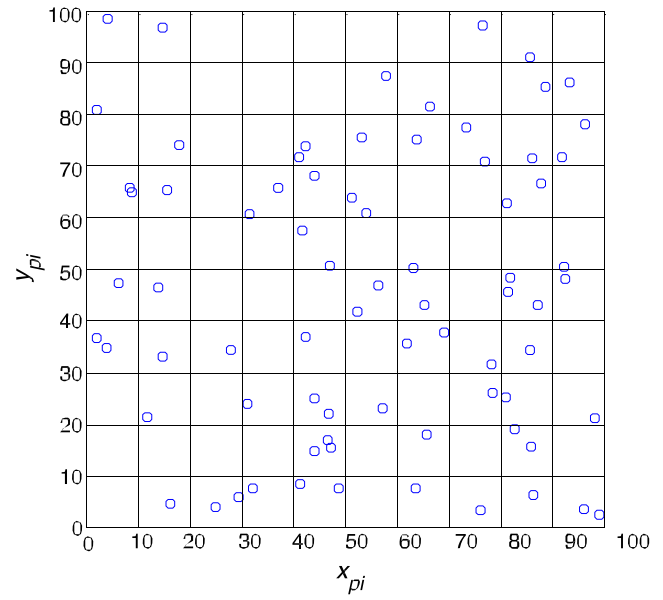

Fig. 1 A given random distribution of $n=100$ particles on the xy plane

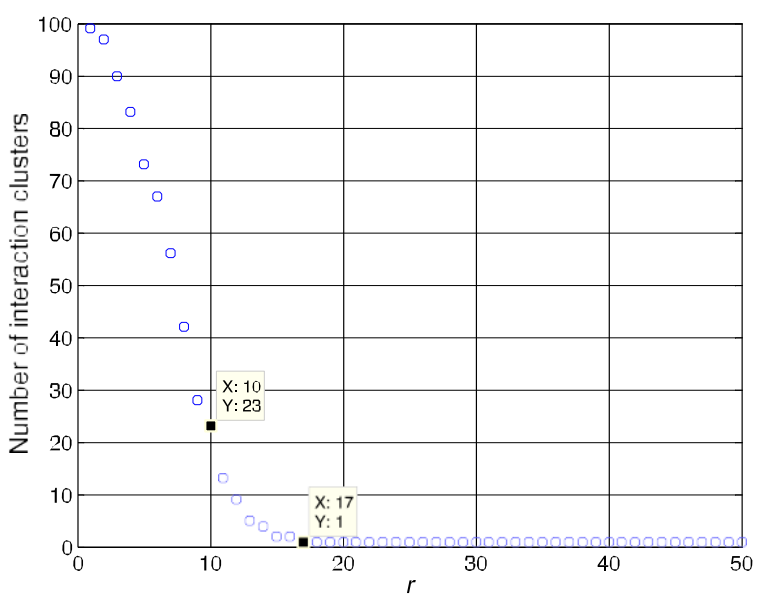

Fig. 2 Number of particles' interaction clusters as a function of the threshold $r$

Figure 2 depicts the corresponding number of particles' interaction clusters as a function of the threshold $r$. It can be seen that, for $r \geq 17$, there is only one interaction cluster, which means that all particles interact with each other.

In such a system, we cannot expect synchronisation between particles that do not interact. Therefore, at most, the system may achieve cluster synchronisation, which means that all particles belonging to the same cluster synchronise. If there is a single cluster, then all particles interact with each other and, consequently, cluster and global synchronisation are equivalent. Global synchronisation can only be expected for this case.

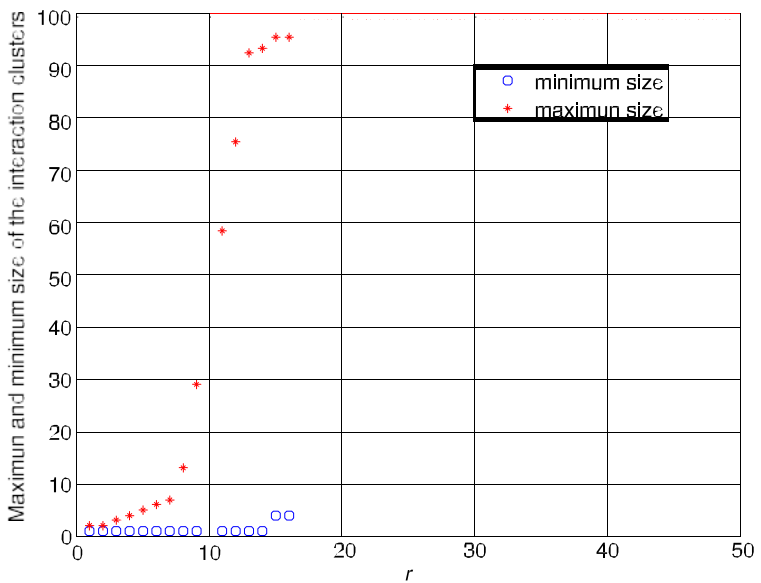

Fig. 3 Maximum and minimum size of the interaction clusters as a function of the threshold $r$

The cluster size also depends on the particles' spatial distribution and threshold $r$. Figure 3 depicts the maximum and minimum size of the interaction clusters, for the particles' distribution of Fig. 1, as a function of the threshold $r$. It can be observed that for $r \geq 17$, as there is only one interaction cluster, the maximum and minimum cluster size is identical and matches the number of existing elemental particles.

In order to analyse the system's behaviour, we compute the discrete Fourier transform (DFT) of the signals $x_{1 \text { sist }}$ and $x_{2 \text { sist, }}$ previously defined in (2) and (3). The DFTs' magnitude is given by

$$
\begin{aligned}
& \left|X_{1 \text { sist }}\left(\omega_{k}\right)\right|=\frac{1}{N} \cdot\left|\sum_{q=0}^{N-1} x_{1 \mathrm{sist}}\left(t_{q}\right) \cdot e^{-j \omega_{k} t_{q}}\right| \\
& \left|X_{2 \operatorname{sist}}\left(\omega_{k}\right)\right|=\frac{1}{N} \cdot\left|\sum_{q=0}^{N-1} x_{2 \operatorname{sist}}\left(t_{q}\right) \cdot e^{-j \omega_{k} t_{q}}\right|
\end{aligned}
$$

where $N=T_{w} / d t$ represents the length of signals $x_{1 \text { sist }}$ and $x_{2 \text { sist }}$, sampled with a period $d t, \omega_{k}=$ $k 2 \pi /(N \cdot d t), t_{q}=q \cdot d t, k=0, \ldots, N-1, q=$ $0, \ldots, N-1$.

Figure 4 shows the DFTs' magnitude as a function of the angular frequency, $\omega k$, and threshold, $r$, for the signals $x_{1 \text { sist }}$ and $x_{2 \text { sist }}$, respectively. It is clear in Fig. 4 that, for larger values of $r$, there exist a single synchronisation cluster. That is, all elemental particles interact with each other and oscillate at the same frequency.

Figure 5 shows contour plots that complement the previous conclusion. For a threshold distance, $r<17$, 


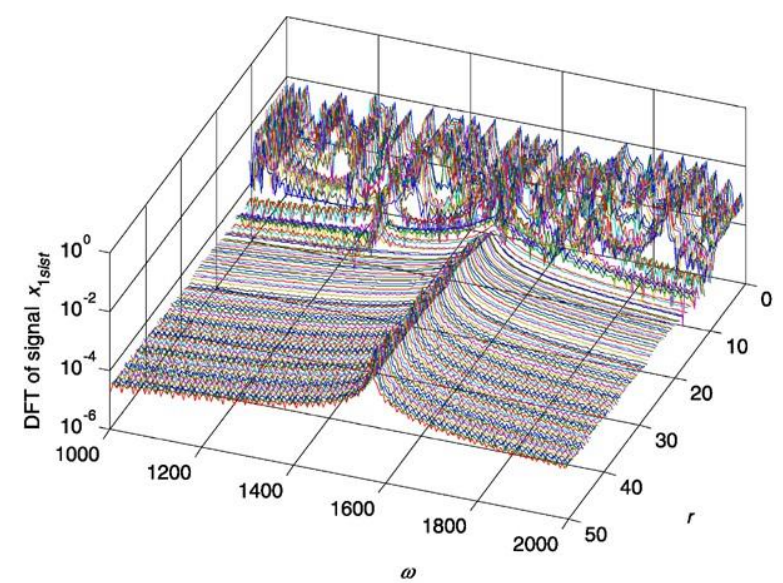

(a)

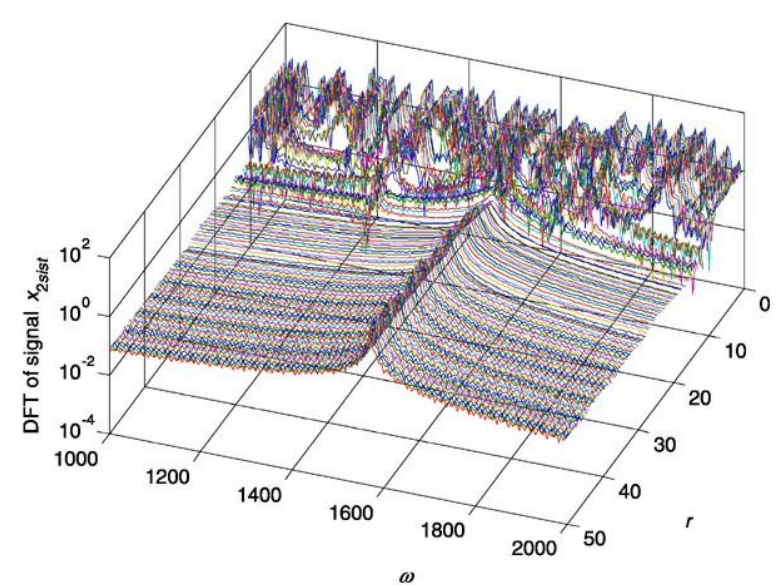

(b)

Fig. 4 Magnitude of the DFT of the signal (a) $x_{1 \text { sist }}$ and (b) $x_{2 \text { sist }}$, as a function of the frequency, $\omega_{k}$, and the distance threshold, $r$

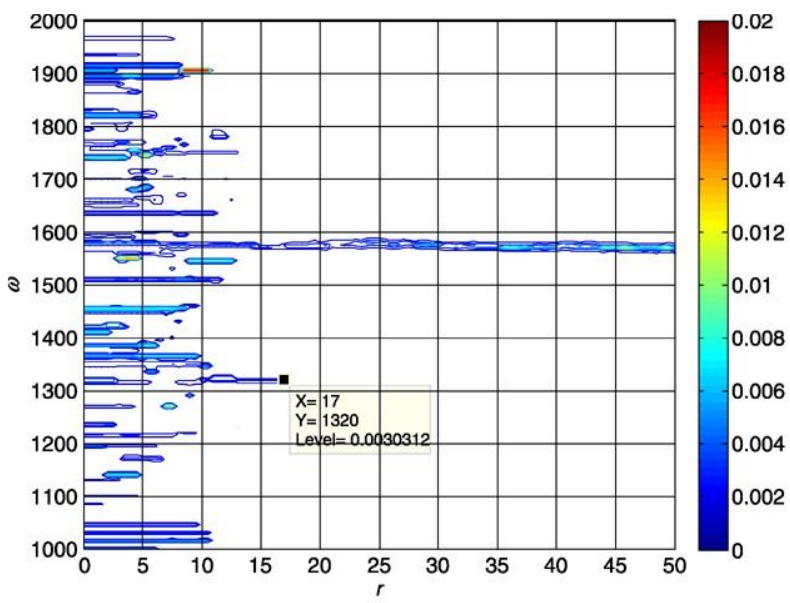

(a)

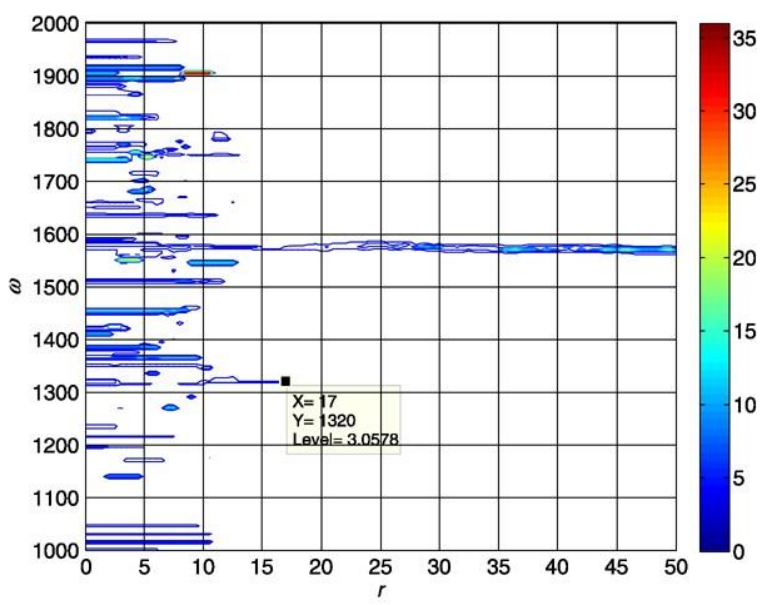

(b)

Fig. 5 Contour plot of the magnitude of the DFTs for the signal (a) $x_{1 \text { sist }}$ and (b) $x_{2 \text { sist }}$, as a function of the frequency, $\omega_{k}$, and the distance threshold, $r$

we notice the appearance of several distinct synchronisation clusters, oscillating synchronously. In each case, the cluster synchronous frequency is given by the arithmetic average of the frequencies that the elemental particles belonging to the cluster would have if they had an independent dynamics. Moreover, it can be noticed that close to the global synchronisation threshold, $r=17$, the particles' amplitude of oscillation is smaller. This means that the global system is dissipative and that, near to the global synchronisation threshold, a minimum energy state is observed.

Synchronisation can also be perceived if we analyse the time evolution of the particles' state variables.
Hence, Figs. 6, 7 and 8 depict illustrative results concerning to variables $x_{1 i}$. An identical behaviour is observed for $x_{2 i}$. Considering that our system is composed of $n=100$ particles and the interaction gain is $G=10000$, the results obtained for three distinct values of the threshold distance, $r$, are discussed. In Fig. 6 the threshold is $r=15$, which means that there are three interaction clusters, as described in Fig. 2. Therefore, cluster synchronisation occurs, as illustrated by the three curves depicted in Fig. 6b. For $r=25$ and $r=50$ a single cluster exists, as discussed before. Figure 7 illustrates these two cases. The single curve that is observed underlines the existence of global synchro- 


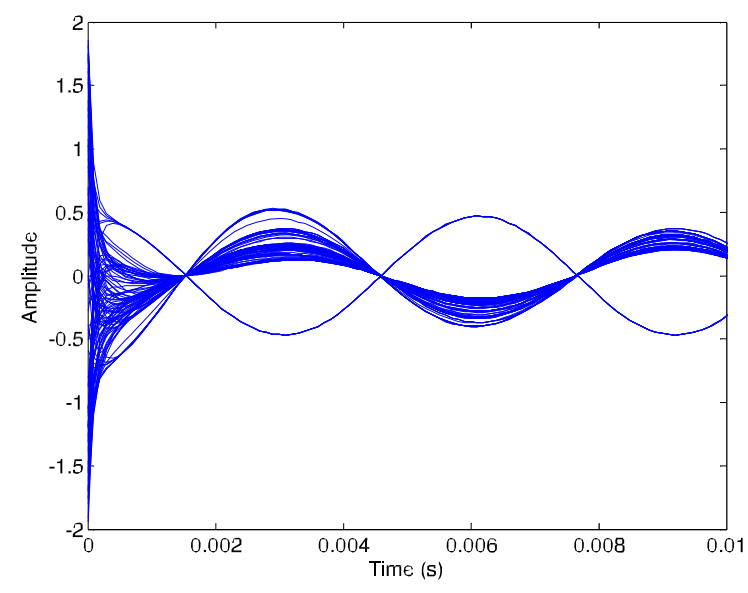

(a)

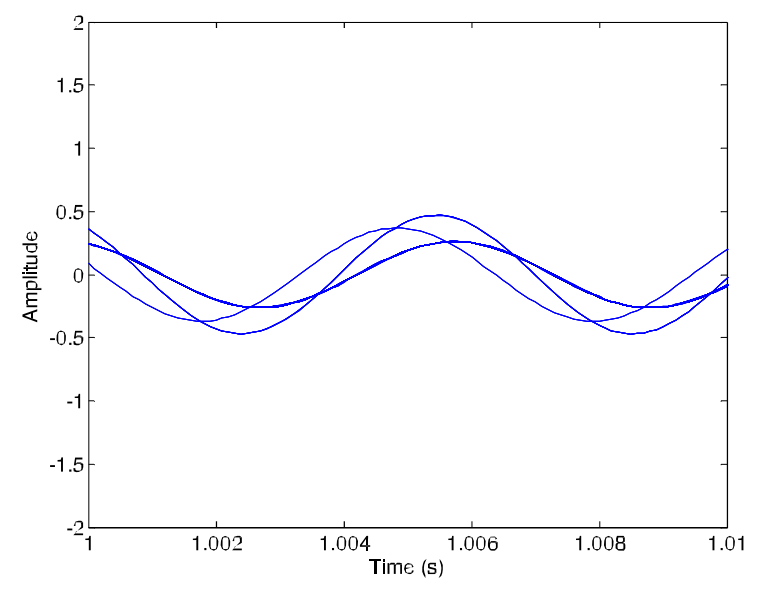

(b)

Fig. 6 Time evolution of the particles' state variables $x_{1 i}$ (threshold $r=15$, number of particles $n=100$, interaction gain $G=10000$ ); (a) time interval $0-0.01 \mathrm{~s}$; (b) time interval $1-1.01 \mathrm{~s}$

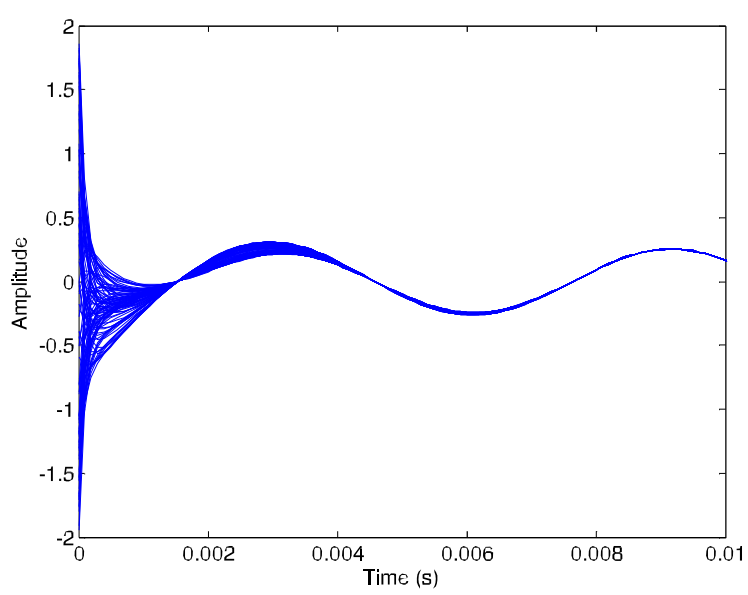

(a)

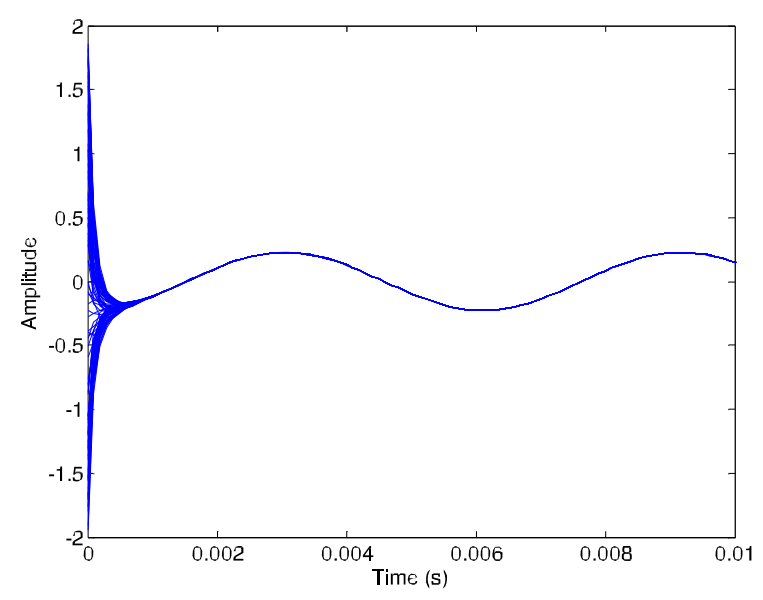

(b)

Fig. 7 Time evolution of the particles' state variables $x_{1 i}$ (number of particles $n=100$, interaction gain $G=10000$ ); (a) threshold $r=25 ;(\mathbf{b})$ threshold $r=50$

nisation in both cases. Moreover, faster synchronisation is possible if $r$ is larger. Choosing the threshold distance $r=50$ and varying the interaction gain, the results of Fig. 8 are obtained. Namely, faster synchronisation is achieved for higher values of the interaction gain.

Additional experiments also revealed that the formation of synchronisation clusters is almost independent of the interaction gain. This means that if all

conditions are unchanged, identical interaction clusters are obtained, independently of the gain. The main difference is on the larger synchronisation time needed if smaller gains are used. This is also illustrated in Fig. 8.

The DFTs' magnitudes yield only information about the frequency. Therefore, two additional measures are introduced and analysed, in order to better characterise the global system. Firstly, the root mean square distances between particles' state variables, $E_{1}$ and $E_{2}$, respectively, are defined:

$$
E_{1}=\sqrt{\frac{1}{N} \cdot \frac{1}{P} \cdot \sum_{q=1}^{N} \sum_{i=1}^{n-1} \sum_{j=i+1}^{n}\left[x_{1 i}\left(t_{q}\right)-x_{1 j}\left(t_{q}\right)\right]^{2}}
$$




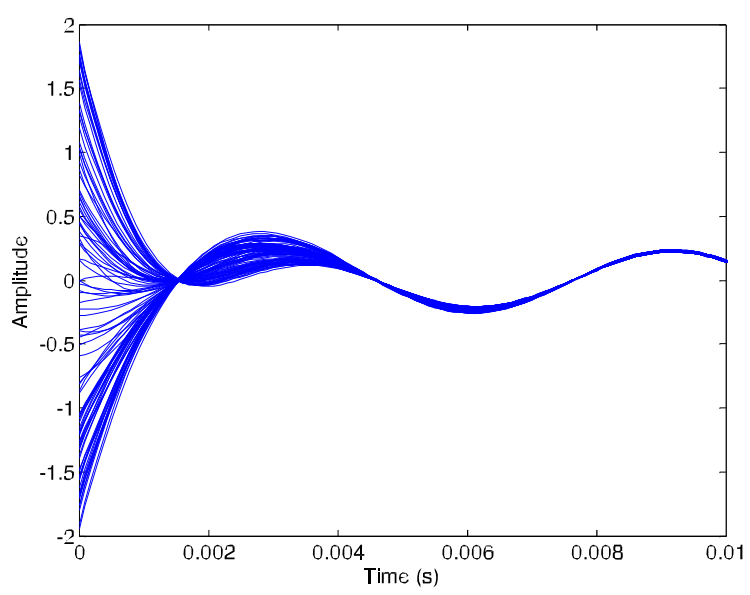

(a)

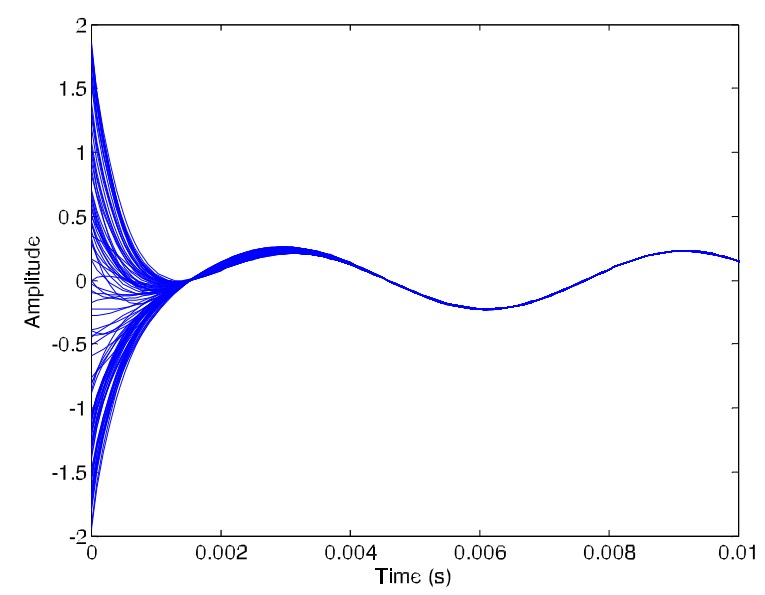

(b)

Fig. 8 Time evolution of the particles' state variables $x_{1 i}$ (threshold $r=50$, number of particles $n=100$ ); (a) interaction gain $G=1000 ;(\mathbf{b})$ interaction gain $G=2000$

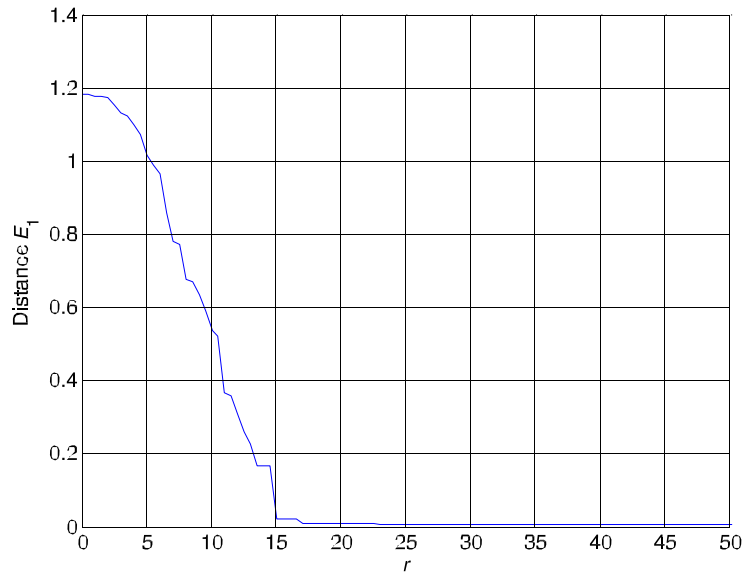

(a)

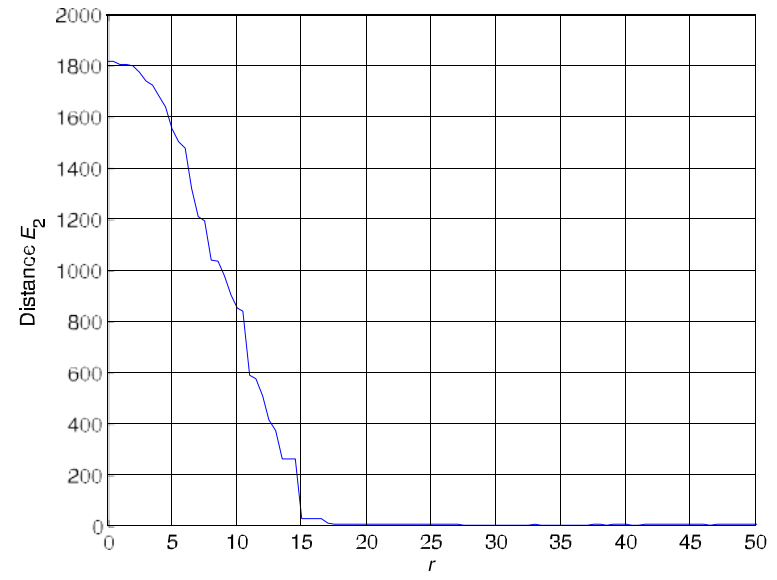

(b)

Fig. 9 Magnitude of the distances (a) $E_{1}$ and (b) $E_{2}$, as a function of the threshold, $r$

$$
\begin{aligned}
& E_{2}=\sqrt{\frac{1}{N} \cdot \frac{1}{P} \cdot \sum_{q=1}^{N} \sum_{i=1}^{n-1} \sum_{j=i+1}^{n}\left[x_{2 i}\left(t_{q}\right)-x_{2 j}\left(t_{q}\right)\right]^{2}} \\
& P=n(n-1) / 2
\end{aligned}
$$

Secondly, the distances between the state variables for all $P$ pairs of elemental particles, at a given time instant, $t_{k}$, are considered. If global synchronisation exists at that time instant, the distances between the corresponding state variables of all pairs of elemental particles are zero, as all particles are in the same state. On the other hand, if cluster synchronisation is verified, only a certain number of distances will be zero. Mathematically, these distances, defined for particles' state

variables, are given by

$$
\begin{aligned}
D_{1} & =\sqrt{\left[x_{1 i}\left(t_{k}\right)-x_{1 j}\left(t_{k}\right)\right]^{2}} \\
i & =1, \ldots, n-1 ; j=i+1, \ldots, n \\
D_{2} & =\sqrt{\left[x_{2 i}\left(t_{k}\right)-x_{2 j}\left(t_{k}\right)\right]^{2}} \\
i & =1, \ldots, n-1 ; j=i+1, \ldots, n
\end{aligned}
$$

Figure 9 shows the distances, $E_{1}$ and $E_{2}$, as a function of the threshold, $r$. It can be seen that the distances decrease with the threshold and almost vanish at $r=17$, 


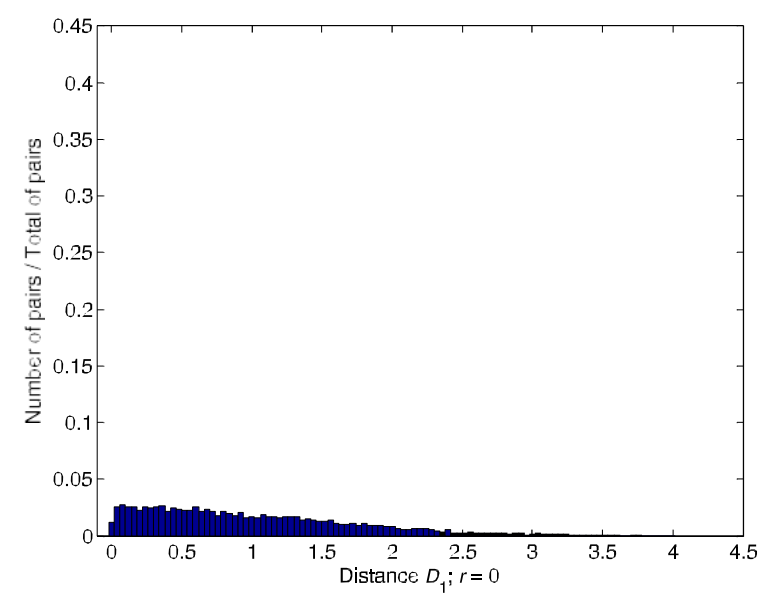

(a)

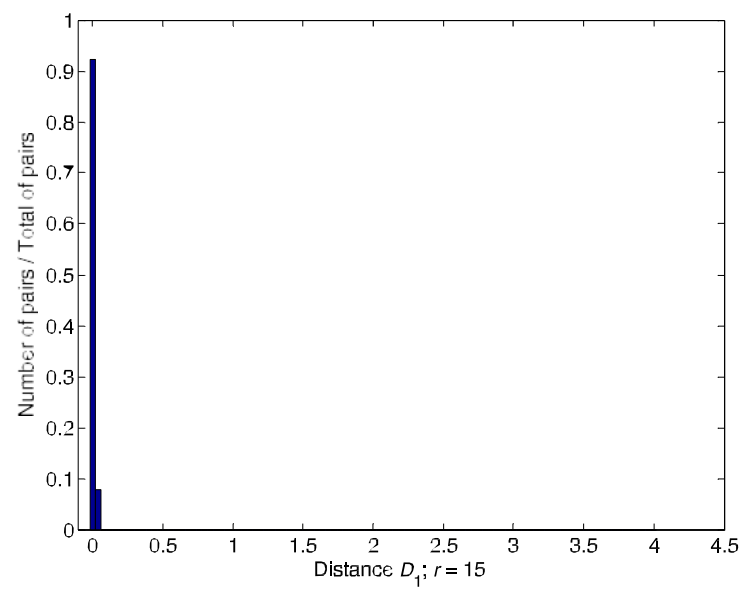

(c)

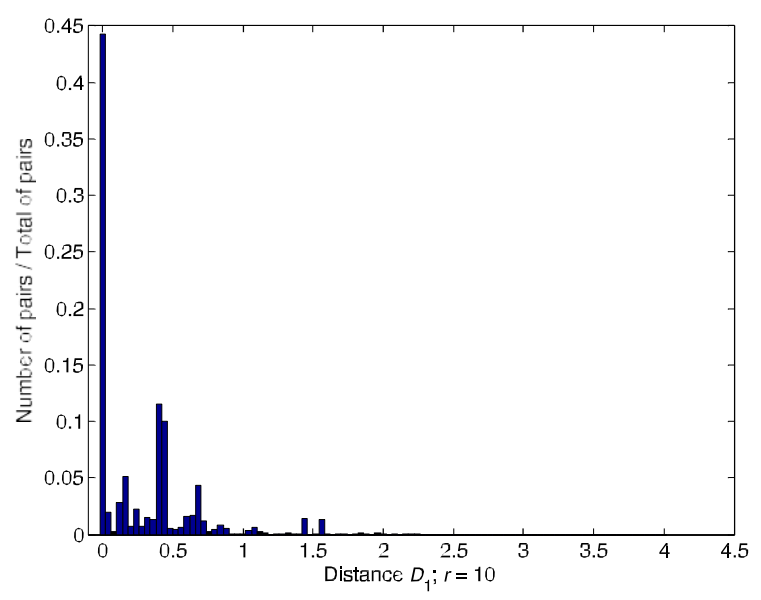

(b)

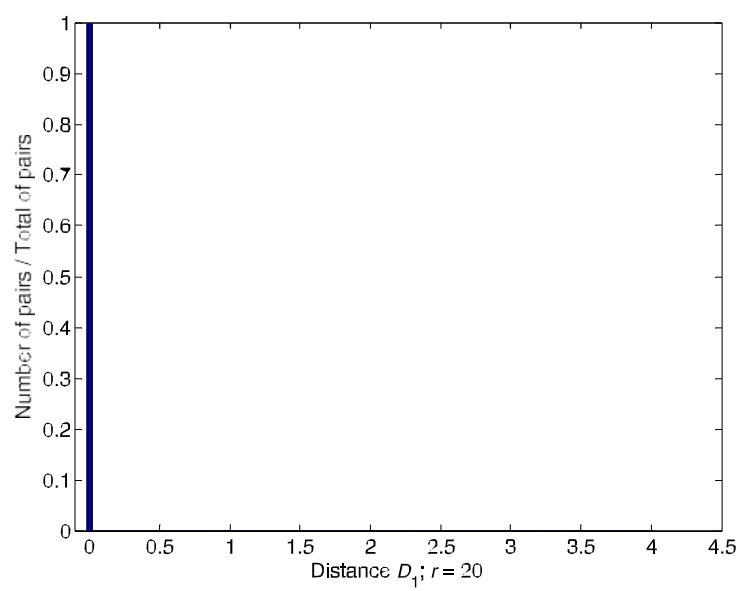

(d)

Fig. 10 Normalised histograms of the distances $D_{1}$, at instant $t_{k}=1 \mathrm{~s}$, for (a) $r=0$, (b) $r=10$, (c) $r=15$, and (d) $r=20$

which means that global synchronisation is achieved. This complements the results of Figs. 4 and 5. In fact the analysis of $E_{1}$ and $E_{2}$ lead to the conclusion that, not only frequency synchronisation can be achieved, but also global synchronisation is possible.

Normalised histograms of distance $D_{1}$, at time instant $t_{k}=1 \mathrm{~s}$, for $r=0, r=10, r=15$ and $r=20$ are represented in Fig. 10. The chosen $t_{k}$ is enough to allow synchronisation and the four increasing values of $r$ represent different interaction conditions. For each case, $D_{1}$ was calculated and the available results were binned into several equally spaced containers. At $r=0$, that is, for no interaction between particles, the non-zero distances between pairs of particles are significant and spread along a large interval (a). As the threshold, $r$, decreases, the distances concentrate at lower values ( $b$ and c) and, at $r=20$, all distances have values near zero (d). These results demonstrate that global synchronisation was achieved. Identical results can be found for distances $D_{2}$, corresponding to the second state variable.

In order to have a deeper understanding of the system behaviour, the distances $E_{1}$ and $E_{2}$ are analysed with respect to the number of elemental particles in the global system, $n$, and the interaction gain $G$ (Figs. 11 and 12). In the former case, $n$ varies from 0 to 100 particles and the threshold distance, $r$, varies between 0 and 50 (as in previous simulations). Analysing the results plotted in Fig. 11, it can be noticed that for higher values of $n$ the limit threshold required to achieve dis- 


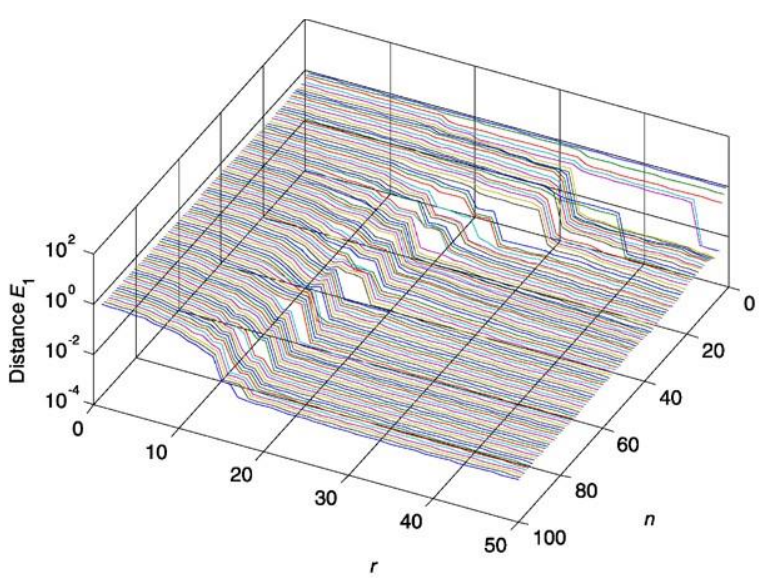

(a)

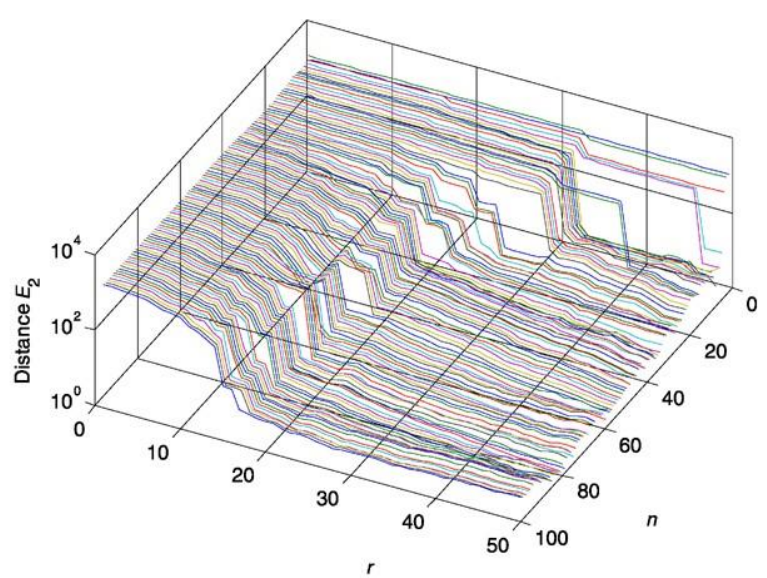

(b)

Fig. 11 Magnitude of the distances (a) $E_{1}$ and (b) $E_{2}$, as a function of the threshold, $r$, and the number of particles, $n$

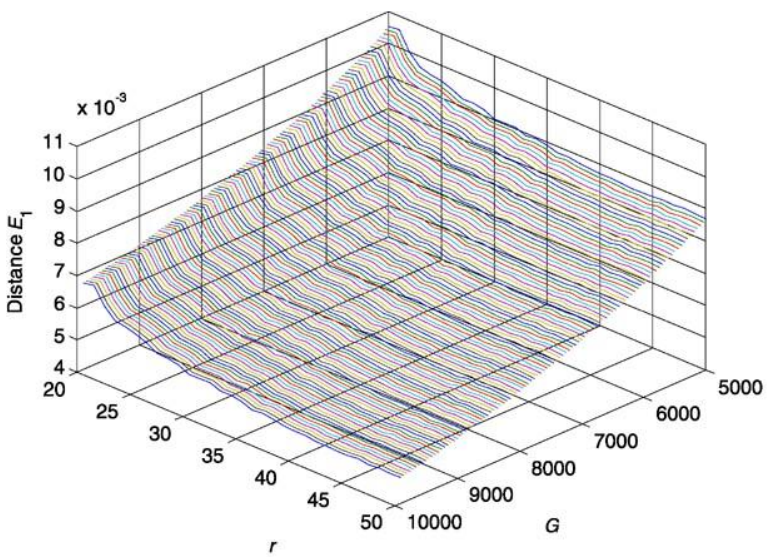

(a)

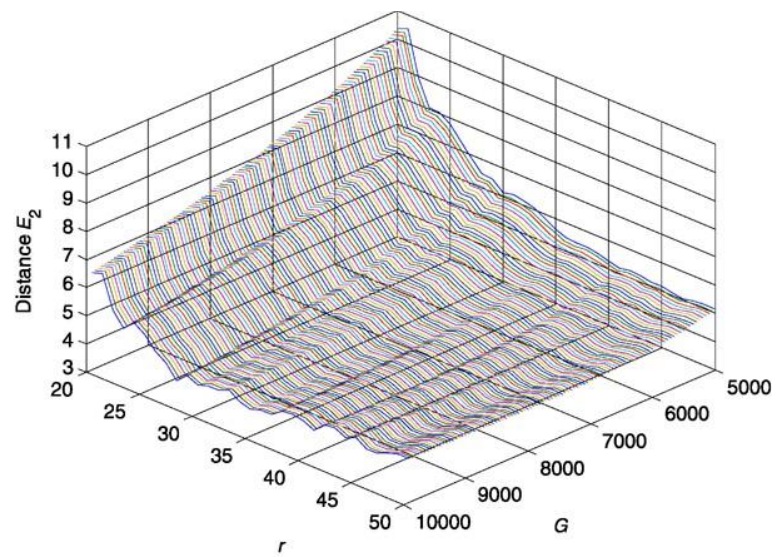

(b)

Fig. 12 Magnitude of the distances (a) $E_{1}$ and (b) $E_{2}$, as a function of the threshold, $r$, and the interaction gain, $G$

tances $E_{1}$ and $E_{2}$ close to zero and, consequently, to attain global synchronisation, is lower. In the latter case, varying the interaction gain, $G$, between $5 \times 10^{3}$ and $10^{4}$, it can be observed that the distances $E_{1}$ and $E_{2}$ became higher for lower values of $G$ (Fig. 12).

To complement the analysis of the system behaviour, the energy of the signals, $x_{1 \text { sist }}$ and $x_{2 \text { sist }}$, that represent the first and second state variables' averages, is analysed. The energy is defined by:

$$
W_{\text {lsist }}=\frac{1}{N} \sum_{k=0}^{N-1}\left|X_{1 \text { sist }}\left(\omega_{k}\right)\right|^{2}
$$

$$
W_{2 \text { sist }}=\frac{1}{N} \sum_{k=0}^{N-1}\left|X_{2 \text { sist }}\left(\omega_{k}\right)\right|^{2}
$$

where $X_{1 \text { sist }}$ and $X_{2 \text { sist }}$ represent the DFT of the signals $x_{1 \text { sist }}$ and $x_{2 \text { sist }}$.

Figures 13 and 14 show the energies $W_{1 \text { sist }}$ and $W_{2 \text { sist }}$ as a function of the threshold distance, $r$, respectively. It can be observed that close to the global synchronisation threshold, $r=17$, the system energy is close to zero. This means that the global system is dissipative and a minimum energy state is observed near to the globat synchronisation condition.

spect to the number of elemental particles, $n$, and inter- 


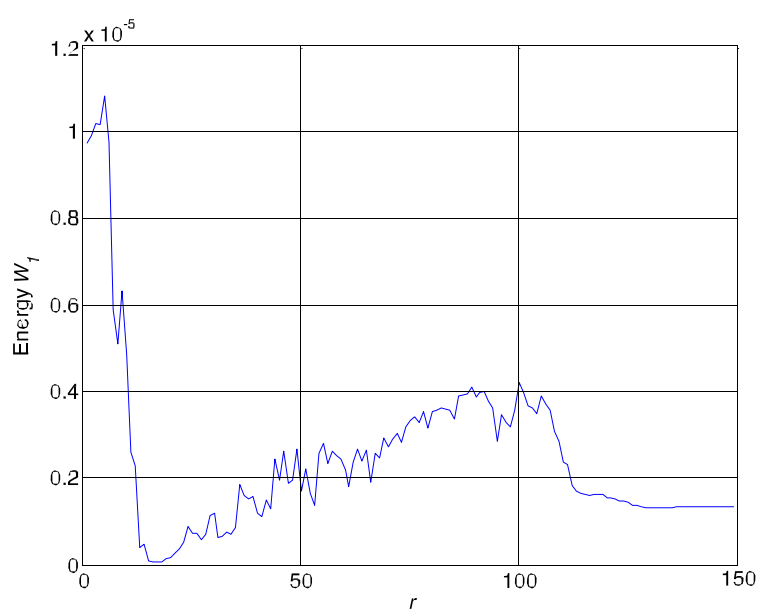

(a)

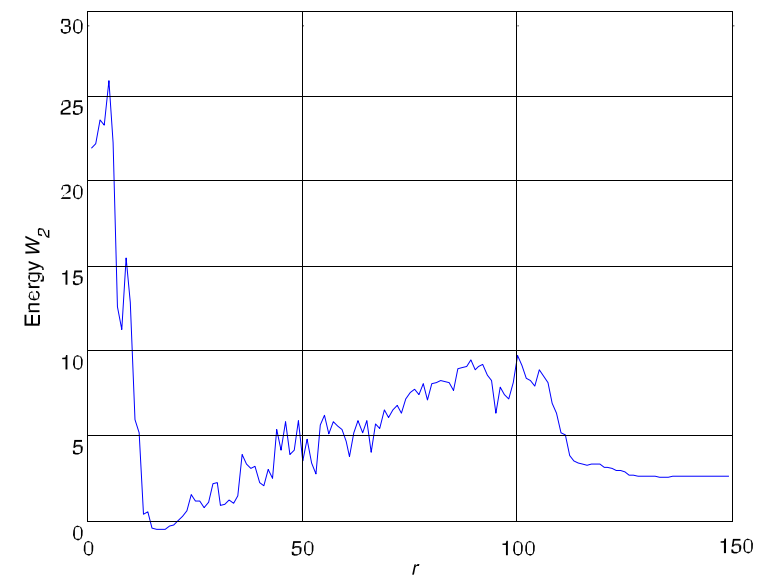

(b)

Fig. 13 Energies (a) $W_{1 \text { sist }}$ and (b) $W_{2 \text { sist }}$, as a function of the threshold, $r$

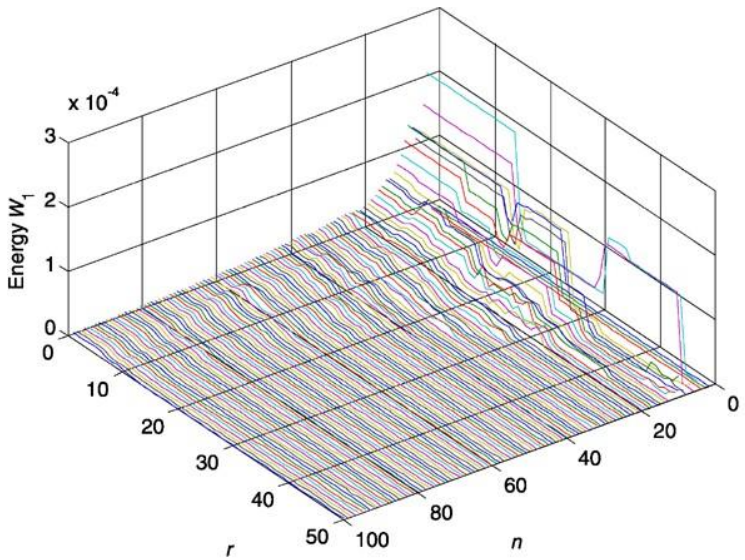

(a)

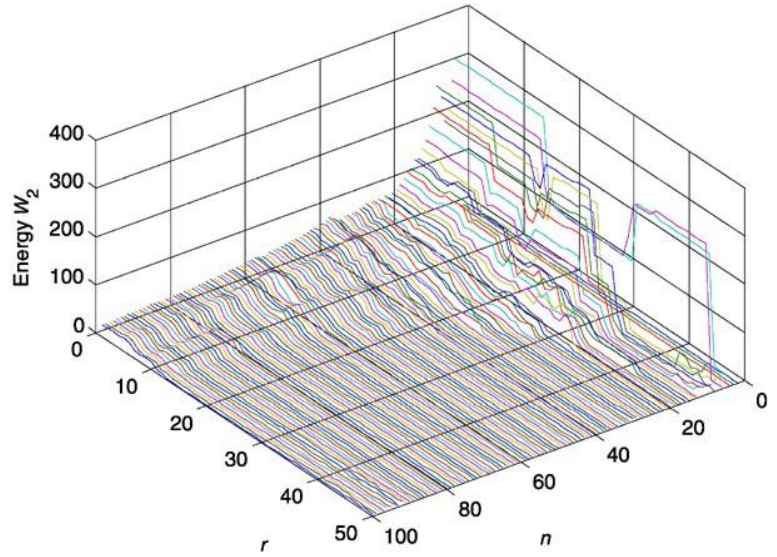

(b)

Fig. 14 Energies (a) $W_{1 \text { sist }}$ and (b) $W_{2 \text { sist }}$, as a function of the threshold, $r$, and the number of particles, $n$

action gain, $G$. Several results are plotted in Figs. 14 and 15. From Fig. 13, it can be seen that, for a very small number of particles, the system energy is somewhat noisy. Then, as the number of particles grows higher, meaning that the interaction gets stronger, more dissipative the system becomes. On the other hand, analysing Fig. 15, it can be noticed that the energy increases with the interaction gain, $G$.

Finally, the time evolution of the total mechanical energy of the system, $E_{\mathrm{KP}}(t)$, is computed and analysed. The total mechanical energy is the sum of the kinetic, $E_{K}(t)$, and the potential, $E_{P}(t)$, energies, given by:

$$
\begin{aligned}
E_{\mathrm{KP}}(t) & =E_{K}(t)+E_{P}(t) \\
& =\frac{1}{2} \sum_{i=1}^{n} M_{i} \cdot x_{2 i}^{2}+\frac{1}{2} \sum_{i=1}^{n} K_{i} \cdot x_{1 i}^{2}
\end{aligned}
$$

The results of the analysis for a given number of elemental particles, $n=100$, and interaction gain, $G=$ $10^{4}$, are depicted in Fig. 16. It can be seen that the energy, $E_{\mathrm{KP}}(t)$, decreases in time whenever synchronisation occurs. Starting with small distance thresholds, $r$, cluster synchronisation is achieved, and the energy diminishes slightly with time. Then, as the threshold approaches the global synchronisation condition, the en- 


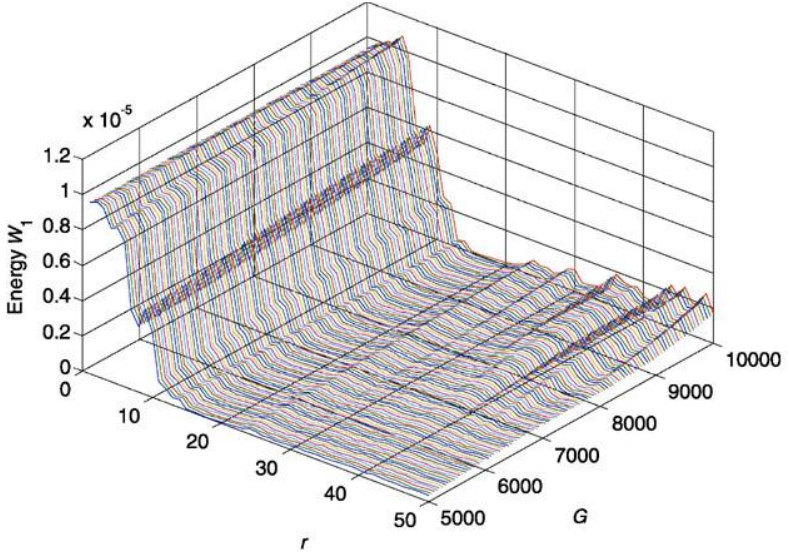

(a)

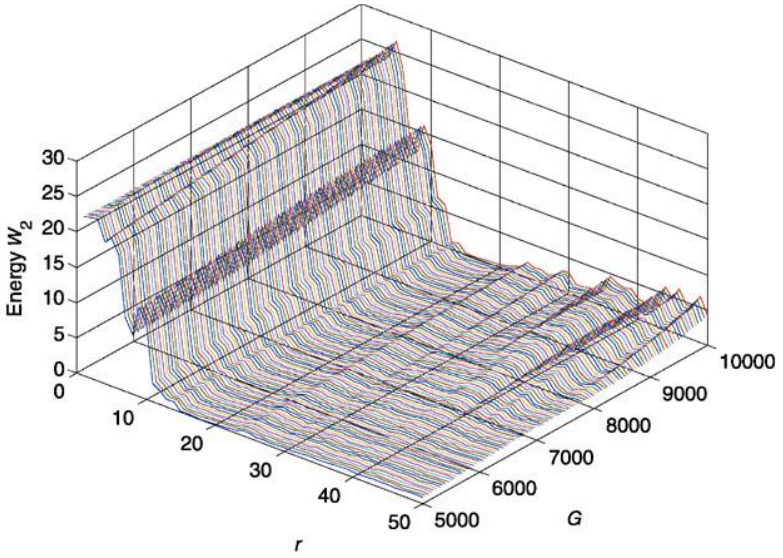

(b)

Fig. 15 Energies (a) $W_{1 \text { sist }}$ and (b) $W_{2 \text { sist }}$, as a function of the threshold, $r$, and the interaction gain, $G$

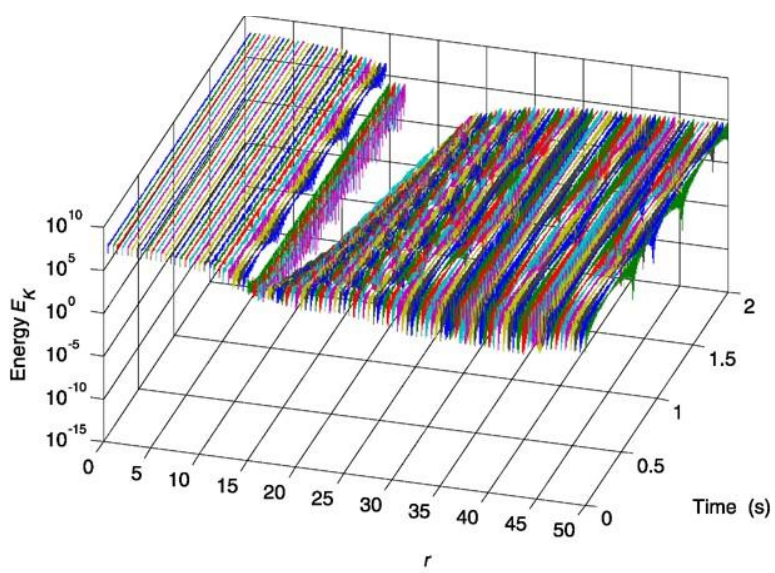

(a)

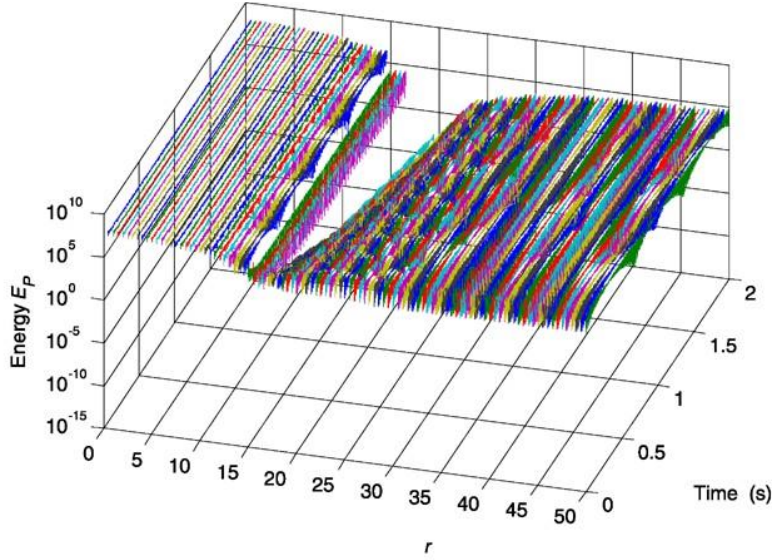

(b)

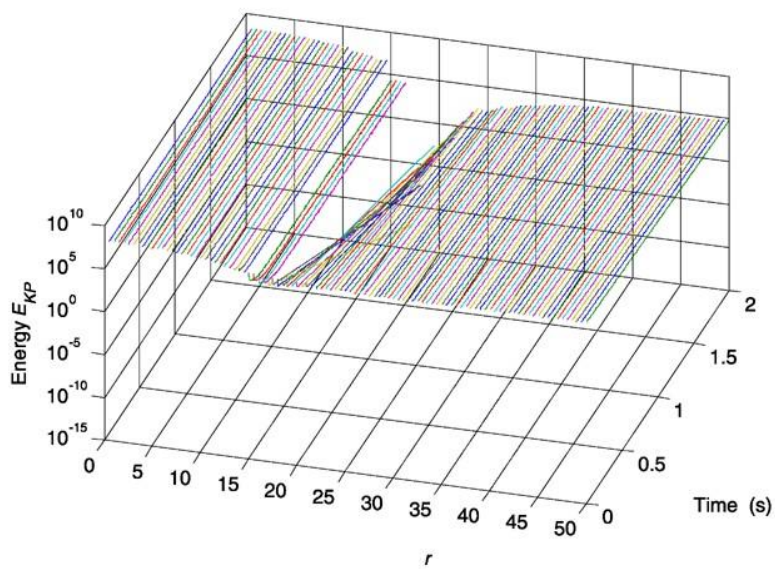

(c)

Fig. 16 System energy: (a) kinetic, $E_{K}(t)$, (b) potential, $E_{P}(t)$, and (c) total mechanical, $E_{\mathrm{KP}}(t)$, as a function of the threshold, $r$, and time, $t$ 
ergy diminishes significantly, drastically falling down when the condition is met. Afterwards, dissipation gets weaker. Similar results are obtained for distinct values of $n$ and $G$.

\section{Conclusion}

Dynamical systems, assembled by a large number of interacting elements, often exhibit collective behaviours. Synchronisation is a particular collective behaviour that may be interpreted as a process by which the individual elements adjust a given property of their motion to a common pattern. Synchronisation phenomena occur widely in nature and in biology. In engineering and technology, synchronisation plays an important role in several areas, namely, secure communication systems, electric power systems and robotics.

In this paper, we study a large-scale dynamical system, constituted by several identical particles that interact with their neighbours. Each particle is a simple linear oscillator and the interaction is given by a statistical feedback law. It is shown that the global system exhibits two main collective behaviours, namely, partial synchronisation, characterised by the existence of several clusters of synchronised particles, and global synchronisation, where all the elemental particles completely synchronise. Moreover, we show that the system becomes dissipative whenever any type of synchronisation occurs.

\section{References}

1. Banerjee, S., Saha, P., Chowdhury, A.R.: On the application of adaptive control and phase synchronisation in non-linear fluid dynamics. Int. J. Non-Linear Mech. 39, 25-31 (2004)

2. Boccaletti, S., Valladares, D.L.: Characterization of intermittent lag synchronisation. Phys. Rev. E 62, 7497-7500 (2000)

3. Boccalettia, S., Kurths, J., Osipov, G., Valladares, D.L., Zhou, C.S.: The synchronisation of chaotic systems. Phys. Rep. 366, 1-101 (2002)

4. Coelho, L.S., Bernert, D.L.A.: PID control design for chaotic synchronisation using a tribes optimization approach. Chaos Solitons Fractals 42, 634-640 (2009)

5. Couzin, I.D.: Collective minds. Nature 445, 715 (2007)

6. Couzin, I.D., Krause, J.: Self-organization and collective behaviour in vertebrates. Adv. Study Behav. 32, 1-75 (2003)

7. Danino, T., Mondragón-Palomino, O., Tsimring, L., Hasty, J.: A synchronised quorum of genetic clocks. Nature 463, 326-330 (2010)
8. Di Bernardo, D., Signorini, M.G., Cerutti, S.: A model of two nonlinear coupled oscillators for study of heartbeat dynamics. Int. J. Bifurc. Chaos 8, 1975-1985 (1998)

9. Dutta, K.: How birds fly together: the dynamics of flocking. Resonance 15, 1097-1110 (2010)

10. Faria, J.J., Krause, S., Krause, J.: Collective behavior in road crossing pedestrians: the role of social information. Behav. Ecol. 21, 1236-1242 (2010)

11. Femat, R., Solis-Perales, G.: On the chaos synchronisation phenomena. Phys. Lett. A 262, 50-60 (1999)

12. Feng, G., Chen, G.: Adaptive control of discrete-time chaotic systems: a fuzzy control approach. Chaos Solitons Fractals 23, 459-467 (2005)

13. Ghosh, D., Chowdhury, A.R., Saha, P.: Bifurcation continuation, chaos and chaos control in nonlinear Bloch system. Commun. Nonlinear Sci. Numer. Simul. 13, 1461-1471 (2008)

14. Grzybowski, J.M.V., Rafikov, M., Balthazar, J.M.: Synchronisation of the unified chaotic system and application in secure communication. Commun. Nonlinear Sci. Numer. Simul. 14, 2793-2806 (2009)

15. Hadji, H.G.E., Orou, J.B.C., Woafo, P.: Synchronisation dynamics in a ring of four mutually coupled biological systems. Commun. Nonlinear Sci. Numer. Simul. 13, 1361$1372(2008)$

16. Han, Q., Hao, L., Zhang, H., Wen, B.: Achievement of chaotic synchronisation trajectories of master-slave manipulators with feedback control strategy. Acta Mech. Sin. 26, 433-439 (2010)

17. Huang, L.L., Feng, R.P., Wang, M.: Synchronisation of chaotic systems via nonlinear control. Phys. Lett. A 320, 271-275 (2004)

18. Kocarev, L., Parlitz, U.: General approach for chaotic synchronisation with applications to communications. Phys. Rev. Lett. 74, 5028-5031 (1995)

19. Kunz, H., Hemelrijk, C.K.: Artificial fish schools: collective effects of school size, body size, and body form. Artif. Life 9, 237-253 (2003)

20. Lin, W., He, Y.B.: Complete synchronisation of the noiseperturbed Chua's circuits. Chaos 15, 023705 (2005)

21. Lu, J., Wu, X., Lü, J.: Synchronisation of a unified chaotic system and the application in secure communication. Phys. Lett. A 305, 365-370 (2002)

22. Luo, A.C.J.: A theory for synchronisation of dynamical systems. Commun. Nonlinear Sci. Numer. Simul. 14, 19011951 (2009)

23. Ngueuteu, G.S.M., Yamapi, R., Woafo, P.: Effects of higher nonlinearity on the dynamics and synchronisation of two coupled electromechanical devices. Commun. Nonlinear Sci. Numer. Simul. 13, 1213-1240 (2008)

24. Ott, E., Grebogi, C., Yorke, J.A.: Controlling chaos. Phys. Rev. Lett. 64, 1196-1199 (1990)

25. Park, E.H., Zaks, M.A., Kurths, J.: Phase synchronisation in the forced Lorenz system. Phys. Rev. E 60, 6627-6638 (1999)

26. Pecora, L.M., Carroll, T.L.: Synchronisation in chaotic systems. Phys. Rev. Lett. 64, 821-824 (1990)

27. Rosenblum, M.G., Pikovsky, A.S., Kurths, J.: Phase synchronisation of chaotic oscillators. Phys. Rev. Lett. 76, 1804-1807 (1996) 
28. Ruan, J., Li, L.J.: An improved method in synchronisation of chaotic systems. Commun. Nonlinear Sci. Numer. Simul. 3, 140-143 (1998)

29. Strogatz, S., Stewart, I.: Coupled oscillators and biological synchronisation. Sci. Am. 269, 68-74 (1993)

30. Tenreiro Machado, J.A.: Fractional dynamics of a system with particles subjected to impacts. Commun. Nonlinear Sci. Numer. Simul. (2011). doi:10.1016/ j.cnsns.2011.01.019

31. Tenreiro Machado, J.A., Galhano, A.: Fractional dynamics: a statistical perspective. J. Comput. Nonlinear Dyn. 3, 233237 (2008)

32. Toner, J., Tu, Y.: Flocks, herds, and schools: a quantitative theory of flocking. Phys. Rev. E 58, 4828-4858(1998)

33. Xiuchun, L., Xu, W., Li, R.: Chaos synchronisation of the energy resource system. Chaos Solitons Fractals 40, 642652 (2009)
34. Yook, S.-H., Meyer-Ortmanns, H.: Synchronisation of Rössler oscillators on scale-free topologies. Physica A 371, 781-789 (2006)

35. Yoshida, K., Sato, K., Sugamata, A.: Noise-induced synchronisation of uncoupled nonlinear systems. J. Sound Vib. 290, 34-47 (2006)

36. Yu, H.J., Liu, Y.Z.: Chaotic synchronisation based on stability criterion of linear systems. Phys. Lett. A 314, 292 298 (2003)

37. Zaks, M.A., Park, E.-H., Rosenblum, M.G., Kurths, J.: Alternating locking ratios in imperfect phase synchronisation. Phys. Rev. Lett. 82, 4228-4231 (1999)

38. Zhou, S., Li, H., Zhu, Z.: Chaos control and synchronisation in a fractional neuron network system. Chaos Solitons Fractals 36, 973-984 (2008)

39. Zhu, H., Zhou, S.: Chaos and synchronisation of the fractional-order Chua's system. Chaos Solitons Fractals 39, 1595-1603 (2009) 